\title{
Longitudinal Stark Effect in Parabolic Quantum Dots
}

This content has been downloaded from IOPscience. Please scroll down to see the full text. 2001 Jpn. J. Appl. Phys. 402002

(http://iopscience.iop.org/1347-4065/40/3S/2002)

View the table of contents for this issue, or go to the journal homepage for more

Download details:

IP Address: 132.239.1.231

This content was downloaded on 06/09/2015 at 23:11

Please note that terms and conditions apply. 


\title{
Longitudinal Stark Effect in Parabolic Quantum Dots
}

\author{
Ross Rinaldi, Milena DeGiorgi, Massimo DeVittorio, Angelo Melcarne, \\ Paolo Visconti, Roberto Cingolani, Harri LiPSANEN ${ }^{1}$, Markku SOPANEN ${ }^{1}$, \\ T. DRUFVA ${ }^{2}$ and Jukka TULKKI ${ }^{2}$ \\ Unità INFM, Università degli Studi di Lecce, Via Arnesano, 7310 Lecce, Italy \\ ${ }^{1}$ Optoelectronics Laboratory, Helsinky University of Technology, Otakaari 7A P.O.Box 3000, FIN-02015 HUT, Finland \\ ${ }^{2}$ Laboratory of Computational Engineering, Helsinki University of Technology, P.O.Box 9400, FIN-02015 HUT, Finland
}

(Received September 11, 2000; accepted for publication October 18, 2000)

\begin{abstract}
We present an experimental and theoretical investigation of the longitudinal quantum confined Stark effect in strain-induced parabolic InGaAs quantum dots. The electric field is applied in the plane perpendicular to the growth axis (quantum well plane), through a sub-micron gap opened in a metallic contact, which contains just a few dots. The changes in the microphotoluminescence ( $\mu$-PL) spectra were measured versus the bias, at low temperature. Due to the device geometry, the electric field profile in the gate is not constant resulting in an asymmetric Stark effect as a function of the applied field polarity. Calculations of the field profile in the conatct gap and of the distorsion of the parabolic-like potential as a function of the applied field have been made in order to correlate the observed changes in the electro-optical properties of the dots with the carrier spill over and wavefunction modifications under electric field.
\end{abstract}

KEYWORDS: quantum dots, stark effect, microluminescence

\section{Introduction}

Quantum effects in semiconductor quantum dots (QDs) are of considerable contemporary interest, stimulated by the rapid progress in nanometer-scale fabrication technology and sub-micron characterization methods. The electron and hole confinement in zero-dimensional (0D) heterostructures modifies the density of states inducing various effects that are interesting from the viewpoint of fundamental physics as well as device application. ${ }^{1,2)}$ Due to their nonlinear electro-optical properties, a wide variety of QD systems have been studied to improve the performances of optical devices based on the electroabsorption and electrorefraction. ${ }^{3,4)}$ Electroabsorption based on the quantum-confined Stark effect (QCSE) is one of the most efficient processes for making optical modulators. ${ }^{5)}$ The understanding of the electric field induced changes in the optical properties of quantum dots is also very important for the implementation of devices like logic gates for quantum computation. $^{6)}$

By applying the electric field perpendicularly to the growth axis it is possible to observe the longitudinal QCSE induced by the asymmetric modification of the QD lateral potential. In this paper, we present a theoretical and experimental study of the longitudinal QCSE performed on a quantum dot device consisting of two planar metallic contacts with a gap of $800 \mathrm{~nm}$ comprising a few strain induced InGaAs quantum dots. The device is biased in order to induce a planar electric field in the gap containing the dots.

The microphotoluminescence spectra show few "single dot" lines originating from dots lying at different distances from the electrodes. Due to the device geometry, the electric field profile in the gap is not constant resulting in an asymmetric Stark effect as a function of applied field polarity. The calculation of the exact electric field profile allowed us to extract the actual values of the field experienced by the dots standing at different positions in the gap. By tuning the intensity of the external electric field it is possible to gradually quench the emission from the excited states and from the ground state. The theoretical evaluation of the emission spectra of the sin- gle parabolic quantum dots as a function of the electric field allowed us to reproduce the observed effects in the optical spectra.

Such a device could be useful for the implementation of a quantum computation gate based on quantum dots, due to the coexistence of few dots experiencing different fields in the devices. In this case the dots have slightly different wavefunctions modified by the local electric field for each applied external bias, and could be driven in parallel by laser beams with slightly different energies resonant to the dot lines to implement a quantum gate operation. ${ }^{7)}$

\section{Experimental}

The samples were grown by metal-organic vapor phase epitaxy at atmospheric pressure and at $635^{\circ} \mathrm{C}$ on a $\mathrm{n}^{+}$ (100)-GaAs substrate. The quantum dots consisted of an $8 \mathrm{~nm} \mathrm{In}_{x} \mathrm{Ga}_{1-x}$ As $(x=0.20)$ quantum well buried by a non-intentionally doped GaAs top and down barriers, of thickness $5 \mathrm{~nm}$ and $100 \mathrm{~nm}$, respectively. Self-organized InP islands (stressors) were grown on the GaAs surface in order to induce a suitable strain potential that laterally confines the carriers in the $\operatorname{In}_{x} \mathrm{Ga}_{1-x}$ As quantum well underneath the stressors. The density of InP stressors is of the order of $10^{9} / \mathrm{cm}^{2}{ }^{8}$ )

Two contact pads connected by a bridge were fabricated by optical lithography and thermal evaporation of $\mathrm{Au}$ on the sample surface. Atomic force microscopy (AFM) lithography was used to open the sub-micron gap on the Au-channel connecting the contacts, whilst all the dots outside the butterfly region were removed by wet etching. The gap between the two gold electrodes was of $800 \mathrm{~nm}$ wide and $3 \mu \mathrm{m}$ long, containing about 30 dots (10 dots per $\mu \mathrm{m}$ of length). The inset of Fig. 1 shows the AFM micrograph of a portion of the gap (about $1.5 \mu \mathrm{m}$ long), where we label 9 quantum dots by capital letters. In the specific part of sample imaged in the inset of Fig. 1, most of the dots are found to be closer to the left contact, whereas only few dots fall at the center of the gap. As discussed later, due to the actual field profile in the gap, the dots located near the contacts experience a stronger elec- 


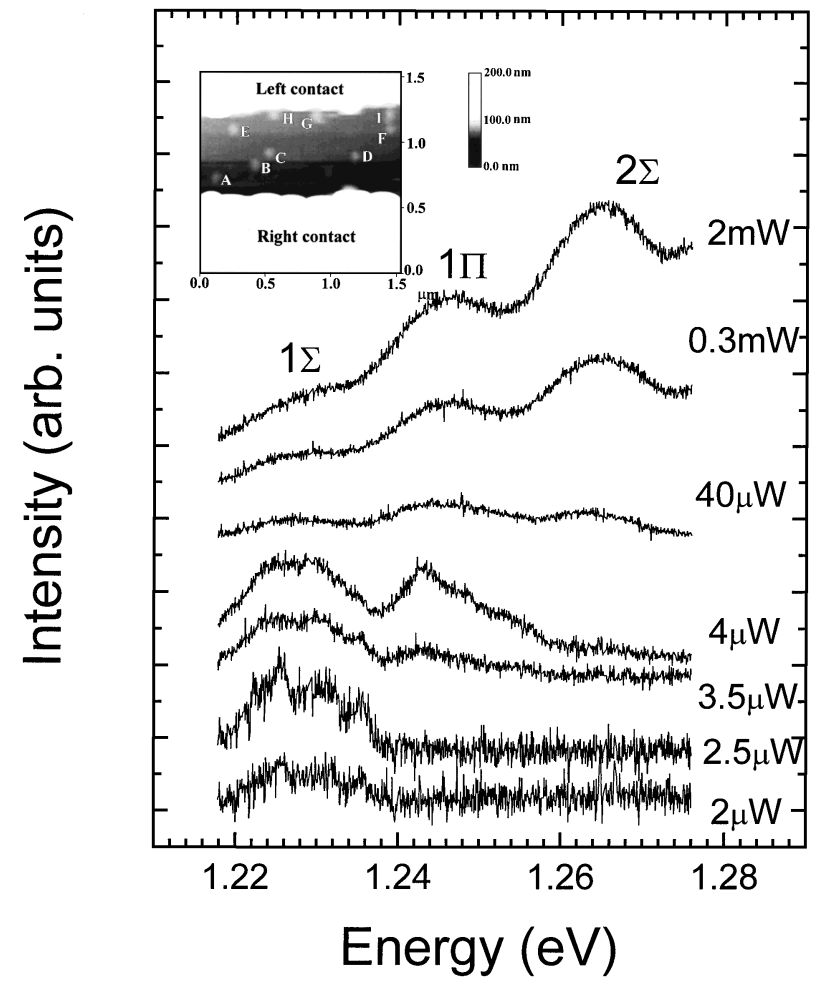

Fig. 1. Intensity dependent $\mu \mathrm{PL}$ spectra of few dots in the gap at zero bias. Inset-AFM micrograph of a portion of the gap containing 9 dots.

tric field. Since the focused laser spot has a diameter of about half a micron, on average we excite three-four dots whose Stark shift depends on their position within the gap.

In our $\mu$-photoluminescence ( $\mu$-PL) set-up the laser probe is focused on the sample surface by a long working distance microscope objective and the photoluminescence of the sample is collected in backscattering mode by the same objective. By using the $514 \mathrm{~nm}$ Ion Argon laser line the focused spot size is of the order of $500 \mathrm{~nm}$, which corresponds to the overall system resolution. The size of the gate was purposely set to $800 \mathrm{~nm}$ in order to be larger than the $\mu \mathrm{PL}$ spot size, but small enough to have a sizeable in-plane field. Both the optical laser pump and the collected signal are coupled to optical fibers. Photoluminescence maps are provided by a motorized $X-Y$ translation stage, that scans the microscope objective over the sample surface. The PL emitted from the dots in the gap was focused on the entrance slits of a single 1-m monochromator and detected by a nitrogen-cooled Ge detector. A range of temperatures between $25 \mathrm{~K}$ and $300 \mathrm{~K}$ is achieved by means of a closed cycle He-Cryostat, modified in order to damp mechanical vibrations.

\section{Results and Discussion}

In Fig. 1 we display the intensity dependent $\mu$-photoluminescence spectra of the dots in the gap at zero bias. At high excitation intensity the spectra resemble the PL spectra collected on the grand ensemble, with the $1 \Sigma$, $1 \Pi$, and $2 \Sigma$ states at energy $1.226 \mathrm{eV}, 1.245 \mathrm{eV}$ and $1.264 \mathrm{eV}$, respectively. The individual linewidth of these bands at $25 \mathrm{~K}$ amounts to about $6 \mathrm{meV}$. As expected, band filling of the excited states occurs at high power, resulting in stronger $1 \Pi$ and $2 \Sigma$ emission bands. By decreasing the excitation power, the excited states are less and less populated, and a

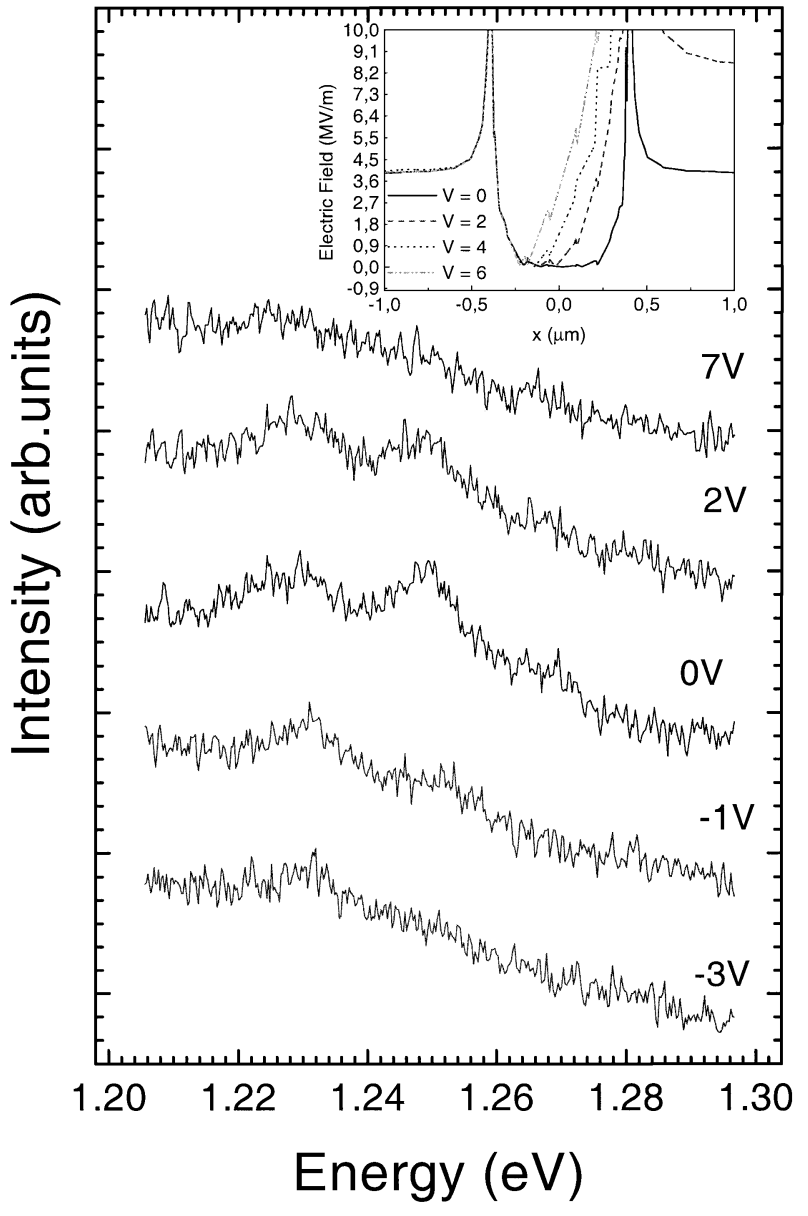

Fig. 2. Photoluminescence spectra of the quantum dots as a function of the applied bias at $25 \mathrm{~K}$. The excitation power density was about $1 \mathrm{~mW}$ and the spot size was $10 \mu \mathrm{m}$. Inset-Calculated electric field profile in the gate as a function of the external bias.

fine structure appears in the emission band of the $1 \Sigma$ band. At the lowest excitation intensity (about $2 \mu \mathrm{W}$ ) four sharp lines (full width at half maximum of about $2 \mathrm{meV}$ ) can be resolved in the envelope of the $1 \Sigma$ band, which are related to four different dots excited in the focused laser spot. The linewidth is limited by the temperature of the experiment $(25 \mathrm{~K})$. The intensity of the emission was rather weak, due to the depletion induced by the Schottky barriers at the edge of the gap.

The PL experiments were repeated with an external electric field applied along the gap between the two electrodes. First of all, we measured the electric field dependent photoluminescence spectra collected from the entire contact gap, by exciting with a spot diameter of about $10 \mu \mathrm{m}$ (Fig. 2). The luminescence spectrum at zero field clearly shows the three bands corresponding to the recombination processes involving respectively the fundamental $1 \Sigma$ state $(1.226 \mathrm{eV})$ and the $1 \Pi$ and $2 \Sigma$ states (at $1.245 \mathrm{eV}$ and $1.265 \mathrm{eV}$, respectively). Under forward bias (left contact positive), we observe the gradual quencing of the second (for biasing voltage of $4 \mathrm{~V}$ ) and first excited states (for biasing voltage of $7 \mathrm{~V}$ ). This is due to the thermionic emission or tunneling processes of carriers in the excited states, which can escape from the parabolic potential well. Our data show that at $7 \mathrm{~V}$, all the dots are depleted, regardless of their position within the contact gap. For reverse bias (left contact negative) the optical spectra show the same 


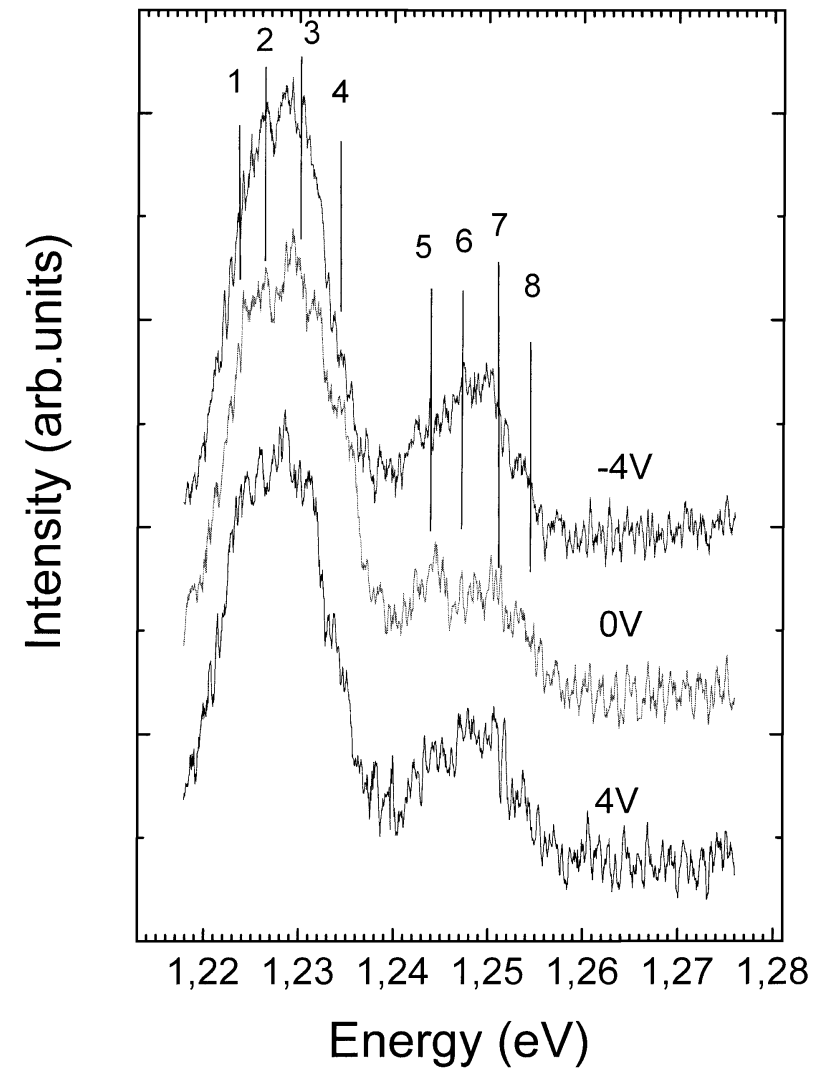

Fig. 3. Microphotoluminescence spectra of the quantum dots as a function of the applied bias. The excitation power density was about $2 \mu \mathrm{W}$ and the spot size was $500 \mathrm{~nm}$. Numbers from 1 to 8 labels the different dot lines, due to the ground state $(1 \Sigma)$ and first excited state $(1 \Pi)$ emission of 4 dots in the spot area.

behavior, although the quenching of the excited states occurs for lower bias $(-3 \mathrm{~V})$. This is consistent with the non uniform distribution of dots in the gap, which are found to be distributed closer to the left electrode than to the right electrode, as shown in the AFM micrograph (inset of Fig. 1). It is important to mention that the measurements were conducted on the same region shown, so that the optical spectra reflects the spatial distribution of dots shown in the inset of Fig. 1. The non uniform spatial distribution of the dots in the nanogate thus enhances the anisotropic behavior of the device in forward and reverse bias due to the electric potential profile. When the spot diameter is reduced to about $0.5 \mu \mathrm{m}$ and the excitation intensity is lowered to few $\mu \mathrm{W}$, the $\mu$-luminescence spectra show different sharp emission lines (Fig. 3) ${ }^{9)}$ corresponding to the recombination processes in the fundamental state and the first excited state of the different "single dots" labelled in the AFM picture.

We note that the $1 \Sigma$ states (labelled with numbers from 1 to 4) do not change much both under reverse and forward bias, with the exception of peak 4 which disappears under reverse bias. The $1 \Pi$ states (labelled with numbers from 5 to 8 ) are found to be more sensitive to the bias, as expected from the larger extent of their wavefunctions. A shift of the order of $1-2 \mathrm{meV}$ is found for the different bands, under bias. Moreover the integrated emission intensities of some of the excited states reduce considerably. As commented above, the extent to which the Stark effect changes the optical spectra of the different dot, depends on the actual field strength experienced by the dots at their specific positions within the gap.

In order to quantify the electric field felt by each dot, and to ascribe the PL lines to the different dots in the inset of Fig. 1, we have to compare these results with the theoretical evaluation of the energy shift of levels and with the calculated electric field profile in the contact gap.

Due to the particular device geometry, the field profile in the sub-micron gap depends on the actual Schottky barriers under the electrodes. To this aim we have numerically solved the Poisson equation for our device structure. The potential induced by the metal-semiconductor Schottky contact propagates in the gap between the two electrodes for about $100 \mathrm{~nm}$ and drops to zero at its center. As a consequence, dots standing close to the contacts can be depleted even at zero bias, resulting in a weakened luminscence efficiency (as indeed observed in the experiments, as compared to the very intense luminescence of the dots without gold contacts). Moreover, without external bias the potential and the electric field profile are perfectly symmetric with respect to the center of the gap. For biases higher than $1 \mathrm{~V}$, the electric field profile in the gap becomes asymmetric (inset of Fig. 2), reaching its maximum near the negative electrode (right contact for forward bias), and decreasing towards the positive electrode. As a consequence the electric field intensity for different bias changes in the gate as a function of the distance from the electrodes. Therefore, dots standing about $100 \mathrm{~nm}$ far from the contacts experience field values exceeding $0.001 \mathrm{~V} / \mathrm{nm}$, already for bias of the order of $1 \mathrm{~V}$, thus being completely depleted. Conversely, dots standing at the center of the gap (300-400 nm far from the contacts) experience typical fields of the order of $0.0001 \mathrm{~V} / \mathrm{nm}$ for a few V bias, so that the Stark effect is negligible. For intermediate distances the Stark effect can be varied by the external bias, and possibly measured by the PL shift and by the variation of the integrated intensity of the emission.

The effect of the in-plane electric field on the quantum dot states have been calculated by using a single band approximation for the electron and heavy-hole wave functions. This has been shown to be a good lowest order approximation due to the reduced heavy-hole-light-hole coupling caused by the compressive strain in the QW. The confinement potential includes the band edge discontinuity, the strain induced deformation potential and the potential of the electric field. The strain induced deformation potential is assumed axially symmetric but the total potential is three dimensional and the eigenfunctions are calculated in 3D. The calculation neglects the piezo electric effect that we assume to be screened due to localization of electrons and holes in spatially separated potential energy minima. The numerical work has been described in ref. 10. The results are given in Fig. 4, where we show the calculated shift in the energy position of the ground and first excited state of the quantum dot. The effect of the electric field on the ground state (Fig. 4(a)) is to produce a shift of the order of $2 \mathrm{meV}$, whereas the first excited state shows a shift of about $2.4 \mathrm{meV}$. The oscillations indicate that the carriers have escaped from the quantum dots, corresponding to the quenched luminescence in the optical spectra. This condition is found to occur at fields of the order of $0.0005 \mathrm{~V} / \mathrm{nm}$. In addition we find that the excited states split into two different states: state described by a wavefunction with a node along the $x$-direction and state $\beta$ described 

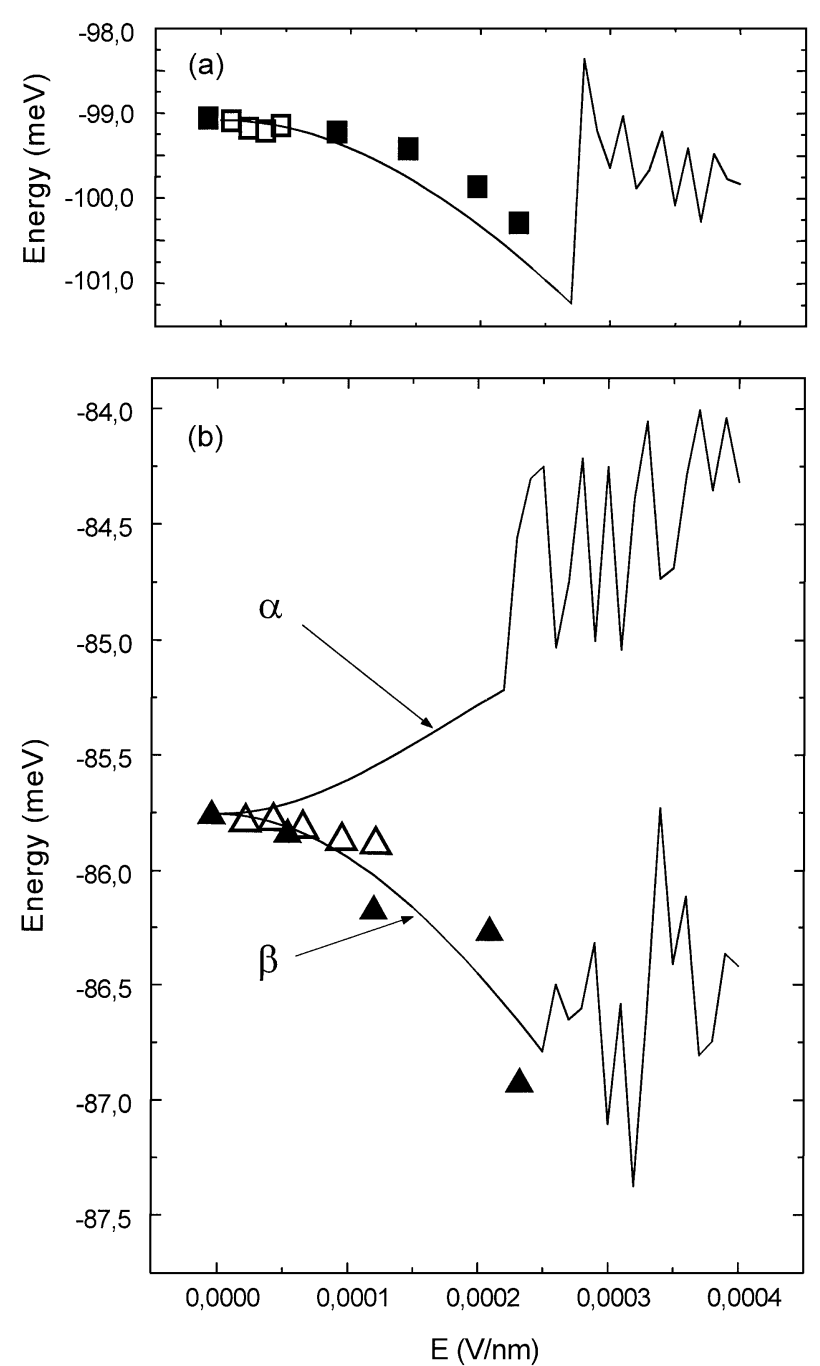

Fig. 4. Theory-Field induced shift (lines) in the electron and hole ground state levels (a) and in the first excited state levels (b). The lines marked by the symbol $\alpha$ and $\beta$ refers to the two splitted states in the first excited levels. The oscillating behaviour at higher field strengths correspond to escape of carriers from the QD. The experimental data (symbols) in the case of forward bias are plotted on the theoretical curves for comparison. The dots showing negligble shift (open symbols) correspond to the dots labelled (E, F) in the inset of Fig. 1, while the dots showing the largest shift (solid symbols) correspond to dots (B, C, D) in inset of Fig. 1.

by a wavefunction with a node along the $y$-direction. These two excited states exhibit different shifts under the external bias (Fig. 4(b)). However, the small splitting among the two states cannot be resolved in our spectra.

The experimental data (symbols) in the case of forward bias are plotted on the theoretical curves for comparison. The dots showing negligble shift (open symbols) correspond to the dots labelled (E, F) in the inset of Fig. 1, which are located about $150 \mathrm{~nm}$ far away from the positive contact (labelled left contact). Conversely, dots (B, C, D), showing the largest shift (solid symbols), are located about $350 \mathrm{~nm}$ far from the left contact, where the electric field varies between 0 and $0.002 \mathrm{~V} / \mathrm{nm}$ when the bias is changed from 1 to $7 \mathrm{~V}$. Though the quantitative agreement between theory and experiment is just indicative, it is important to note that the quench of the emission occurs at the field value consistent with the oscillation shown in Fig. 4(b). Moreover, the behavior of the dots reflects the field profile, thus allowing to distinguish the individual dots according to their position.

\section{Conclusions}

In conclusion we have studied the longitudinal Stark effect in parabolic quantum dots by applying an external electric field perperndicularly to the growth direction. The device geometry and the non uniform dot distribution in the gap between the electrodes result in a asymmetric Stark effect in the elctrooptical properties of the dots.

\section{Acknowledgements}

The expert technical help of D. Cannoletta, I. Tarantini and E. D'Amone is gratefully acknowledged. Part of the work was supported by EC through the "SQID" project within the Future and Emerging Technology programme and by the INFM PRA '99 SSQI.

1) Y. Arakawa and H. Sakaki: Appl. Phys. Lett. 40 (1982) 939.

2) M. Asada, Y. Miyamoto and Y. Suematsu: IEEE J. Quantum Electron. QE-22 (1986) 1915.

3) D. A. B. Miller, D. S. Chemla and S.Schmitt-Rink: Appl. Phys. Lett. 52 (1988) 2154.

4) K. G. Ravikumar, T. Aizawa, K. Matsubara, M. Asada and Y. Suematsu: J. Technol. 9 (1991) 1376.

5) D. A. B. Miller, D. S. Chemla, T. C. Damen, A. C. Gassard, W. Wiegmann, T. H. Wood and C. A. Burrus: Phys. Rev. B 32 (1985) 1043.

6) G. D. Sanders, K. W. Kim and W. C. Holton: Phys. Rev. B 61 (2000) 7526.

7) E. Biolatti, R. C. Iotti, P. Zanardi and F. Rossi: Phys. Rev. Lett. 85 (2000) 5647.

8) H. Lipsanen, M. Sopanen and J. Ahopelto: Phys. Rev. B 51 (1995) 13868.

9) The linewidth of about $2.5 \mathrm{meV}$ is due both to the emission of two or three dots with rather similar dimensions and to the presence of an electric field and interactions with nearby dots. On average 5-6 dots were excited when the spot diameter was of the order of $8 \mu \mathrm{m}$.

10) J. Tulkki and A. Heinanneki: Phys. Rev. B 52 (1995) 8239. 